\title{
Survival Disparities in Bladder, Kidney, and Prostate Cancer in Asian-Indian \& Pakistani Immigrants to the United States as Compared to Other US Ethnic Groups: A Surveillance, Epidemiology and End Result (SEER) 2011 Public Use Database Study
}

\author{
Akm Hossain ${ }^{1}$, Thomas F. Hogan ${ }^{1}$, Rubayat Rahman ${ }^{1}$ \& Gerald Hobbs Jr. ${ }^{1}$ \\ ${ }^{1}$ West Virginia University, Morgantown, United States \\ Correspondence: Thomas F Hogan, Professor of Medicine, West Virginia University, Morgantown, WV 26506, \\ United States. E-mail: thogan@hsc.wvu.edu
}

Received: September 20, 2012 Accepted: October 7, 2012 Online Published: February 19, 2013

doi:10.5539/cco.v2n1p103 URL: http://dx.doi.org/10.5539/cco.v2n1p103

\begin{abstract}
Objective: To compare the SEER 2011 public use data prognostic variables and survival of US Asian-Indians and Pakistanis (US-AIP) diagnosed with bladder, kidney, or prostate cancers, to patients in three comparable US ethnic groups (African-Americans, Hispanics, and Caucasians) diagnosed with the same cancers. Patients and Methods: SEER 1988-to-2008 data (released in 2011) was analyzed using SEER*Stat Software V7.0.5 and SAS version 9.3. Results: From 1988-to-2008, SEER documented 14,006 cancers in US-AIP (7,118 male, 6,888 female), with 2,867 (20\%) being bladder, kidney, or prostate. Compared to the three other US ethnic groups examined, US-AIP genitourinary (GU) cancers occurred at significantly younger ages $(\mathrm{p}<0.01$-to- $<0.001)$. US-AIP had significantly more grade 3-4 $(\mathrm{p}<0.01$-to- $<0.001)$ and stage $3-4(\mathrm{p}<0.001)$ cancers than the other three groups. Paradoxically, despite having worse prognostic factors, five year age-adjusted survival for the bladder, kidney, and prostate cancers in US-AIP were significantly better than in the other three US ethnic groups $(\mathrm{p}<0.01-$ to- $<0.001)$. Conclusions: SEER 1988-to-2008 data contained a "survival disconnect" for genitourinary cancers in US-AIP. All three US-AIP GU cancers had worse prognostic features but better age-adjusted survival when compared to three other US ethnic groups, even though US-AIP appeared to have had similar treatment. US-AIP cultural and biologic factors, which might modify the clinical behavior of their GU cancers, are discussed. Of note, SEER 2000-to-2008 data showed a significant decline in bladder, kidney, and prostate cancer relative risk of death for all four ethnic groups studied versus earlier era SEER 1994-to-2000 data.
\end{abstract}

Keywords: Asian Indian, disparities, epidemiology, Pakistani, SEER, urologic cancer

\section{Abbreviations}

AA: African American

AIP: Asian Indian or Pakistani

DRE: Digital rectal examination

GU: Genitourinary

H: Hispanic

IRB: Institutional Review Board

NCI: National Cancer Institute (United States)

NGO: Non-government organization

NS: Not significant

PSA: Prostate specific antigen

RF: Referent category

SEER: Surveillance, Epidemiology and End Result 


\section{US: United States \\ W: White or Caucasian}

\section{Introduction}

Genetic, environmental, social, and cultural habits may contribute to health disparities. Conversely, identified health disparities may provide insights into disease biology or other factors influencing a given disease.

The SEER (Surveillance, Epidemiology and End Result) program of the United States (US) National Cancer Institute $(\mathrm{NCI})$ is a recognized standard for structure and quality of data for cancer registries. SEER began collecting US cancer data in January 1973, and the latest public use database, covering the 35 years from 1973 to 2008, was released May 2011 ("SEER", 2011). Previously, SEER included racial and ethnic data for major genitourinary (GU) cancers occurring only in African-Americans (AA), Hispanics (H), Chinese, Japanese, and Pacific Islanders, and US Caucasians (W). However, from 1988 onward ("SEER", 2011; Jani et al., 2009), SEER also began collecting cancer incidence in US Asian-Indian and Pakistani (US-AIP) populations.

Because the SEER 2011 data release separately reported 20 years of cancer information for US-AIPs, we had the opportunity to review and compared GU tumor characteristics and survival in US-AIP with bladder, kidney, or prostate cancer to three other US ethnic groups - African Americans, Hispanics, and Caucasians.

\section{Methods}

For US-AIP, we reviewed the available public use SEER data for bladder, kidney, and prostate cancers from 1988 to 2008 (data released in May 2011) which included approximately 3 million patients. Since this review used de-identified NCI public data, it was IRB exempted. Within each patient group we examined age and sex, marital status, tumor stage and grade, methods of confirmation, and treatment modalities (surgery, systemic therapy, or radiation therapy). In SEER, "systemic therapy" did not distinguish between chemotherapy, hormonal therapy, or immunotherapy. The racial or ethnic data in the SEER database has been considered accurate and it is validated by sensitivity studies performed by SEER every even year ("SEER", 2011).

\subsection{Statistical Analysis}

Seer data were summarized in tables listing count and percentage for categorical data and median and quartile data where appropriate. Binary indicators (yes-no) were applied to each type of treatment. Age cut points were chosen for each ethnic group and type of cancer based on the median age at diagnosis. Year 2000 United States census data were used as the population denominators to calculate cancer incidence rates for US-AIP and for the other three ethnic groups studied (“SEER", 2011; Jani et al., 2009; Hossain et al., 2008).

Using SEER*Stat Software V7.0.5 (SEER*stat software V7.0.5, 2011), variables were compared using $t$ tests for continuous variables and chi-square or Fisher's exact tests for categorical variables. Multivariable analysis was performed on age at diagnosis, stage of disease, grade of disease, type of treatment, year of diagnosis, marital status, and SEER region using SAS version 9.3 (SAS v 9.3, 2011), to examine the effect of these variables on outcomes. Relative risk (RR) for death was calculated and compared for age at diagnosis, year of diagnosis, marital status, stage and grade at diagnosis, and treatments received using Cox proportional hazards regression (SAS PHREG). Patients with incomplete data were censored or excluded during subgroup analysis.

Age-adjusted survival analysis by stage and sex was done for each population group using the Kaplan-Meier survival method. US-AIP survival was compared to survival of comparable US African Americans, Hispanics, and Whites/Caucasians who had the same genitourinary cancers. In Tables 1-to-3 the hypothesis tested in the right-most column was that the US-AIP group was not significantly different from the other three ethnic groups combined data. A two-tailed p-value of 0.05 or less was considered statistically significant.

\section{Results}

There were 14,006 US-AIP cancers documented in SEER from 1988-to-2008, 7,118 in men and 6,888 in women. Genitourinary cancer (bladder, kidney, or prostate) accounted for 2,867 (20\%) of all cancers in US-AIP.

\subsection{Bladder Cancer}

Bladder cancer accounted for 417 (3\%) of all cancers in US-AIP in the SEER database. This was similar to the other three US ethnic groups sampled (3\% - to- $4 \%$ ). The US-AIP male/female ratio of bladder cancer at diagnosis was also similar to the other three ethnic groups (Table 1). The calculated incidence rate for bladder cancer in US-AIP (17 per 100,000) was similar to that in African-Americans (20 per 100,000) and US-Hispanics (19 per $100,000)$, but was significantly lower than the incidence rates in US-Whites (40 per 100,000; $<<0.001)$. 
US-AIP men and women were diagnosed with bladder cancer earlier than patients in the other three ethnic groups, and significantly more US-AIPs were diagnosed with higher grade and stage bladder cancer than in the other three ethnic groups.

Table 1. Bladder cancer epidemiology \& survival in US-Asian Indians and Pakistanis (US-AIP) versus other US ethnic groups reported in SEER 1988-2008. Data are presented as number (\%) unless otherwise stated

\begin{tabular}{|c|c|c|c|c|c|}
\hline Variables & US-AIP & US-African & US-Hispanic & US-White & $P$-value \\
\hline SEER Total Cancers & 14,006 & 287,533 & 227,784 & $3,745,846$ & $N A$ \\
\hline BLADDER Cancer & $417(3 \%)$ & $8,626(3 \%)$ & $7,192(3 \%)$ & $149,756(4 \%)$ & $P=0.38$ \\
\hline Incidence $/ 100,000$ & 17 & 20 & 19 & 40 & $<0.001$ \\
\hline Males & $329(79 \%)$ & $6,210(72 \%)$ & $5,537(77 \%)$ & $109,321(73 \%)$ & $P=0.08$ \\
\hline \multicolumn{6}{|l|}{$M+F$ Ages } \\
\hline Median, yrs & 66 & 69 & 72 & 73 & \multirow[t]{3}{*}{$<0.001$} \\
\hline 25th quartile, yrs & 55 & 57 & 61 & 62 & \\
\hline $75^{\text {th }}$ quartile, yrs & 78 & 81 & 83 & 85 & \\
\hline \multicolumn{6}{|l|}{ Male (M) Ages } \\
\hline median, yrs & 65 & 69 & 73 & 73 & \multirow[t]{3}{*}{$<0.001$} \\
\hline 25th quartile, yrs & 54 & 57 & 61 & 61 & \\
\hline 75th quartile, yrs & 78 & 82 & 84 & 86 & \\
\hline \multicolumn{6}{|l|}{ Female (F) Ages } \\
\hline median & 67 & 70 & 71 & 75 & \multirow[t]{3}{*}{$<0.001$} \\
\hline 25th quartile, yrs & 56 & 56 & 62 & 63 & \\
\hline 75th quartile, yrs & 79 & 81 & 83 & 83 & \\
\hline Age $<65$ at diagnosis & $154(37 \%)$ & $2,498(29 \%)$ & $2,013(28 \%)$ & $38,932(26 \%)$ & $<0.01$ \\
\hline Grade I & $96(23 \%)$ & $2,415(28 \%)$ & $2,157(30 \%)$ & $46,420(31 \%)$ & $P \leq 0.01$ \\
\hline Grade II & $137(33 \%)$ & $3,536(41 \%)$ & $2,877(40 \%)$ & $62,819(42 \%)$ & $P \leq 0.01$ \\
\hline Grade III & $104(25 \%)$ & $1,725(20 \%)$ & $1,510(21 \%)$ & $26,903(18 \%)$ & $P \leq 0.01$ \\
\hline Grade IV & $80(19 \%)$ & $949(11 \%)$ & $647(9 \%)$ & $13,521(9 \%)$ & $P \leq 0.001$ \\
\hline Stage I & $88(21 \%)$ & $2,243(26 \%)$ & $2,158(30 \%)$ & $43,493(29 \%)$ & $<0.001$ \\
\hline Stage II & $88(21 \%)$ & $2,588(30 \%)$ & $2,373(33 \%)$ & $46,487(31 \%)$ & $<0.001$ \\
\hline Stage III & $199(48 \%)$ & $3.191(37 \%)$ & $2,085(29 \%)$ & $47,991(32 \%)$ & $<0.001$ \\
\hline Stage IV & $42(10 \%)$ & $604(7 \%)$ & $575(8 \%)$ & $12,049(8 \%)$ & $<0.001$ \\
\hline Surgery & $384(92 \%)$ & $8,195(95 \%)$ & $6,473(90 \%)$ & $140,712(94 \%)$ & $P=0.09$ \\
\hline Radiation & $179(43 \%)$ & $3,795(44 \%)$ & $2,877(40 \%)$ & $56,817(38 \%)$ & $P=0.16$ \\
\hline Systemic therapy & $367(88 \%)$ & $7,763(90 \%)$ & $6,472(90 \%)$ & $127,492(85 \%)$ & $P=0.13$ \\
\hline 1 yr survival & $375(90 \%)$ & $6,642(77 \%)$ & $6,113(85 \%)$ & $122,918(82 \%)$ & $<0.001$ \\
\hline 2 yr survival & $358(86 \%)$ & $6,124(71 \%)$ & $5,681(79 \%)$ & $112,782(75 \%)$ & $<0.001$ \\
\hline 3 yr survival & $341(82 \%)$ & $5,607(65 \%)$ & $5,250(73 \%)$ & $104,629(70 \%)$ & $<0.001$ \\
\hline 4 yr survival & $317(76 \%)$ & $5,262(61 \%)$ & $4,890(68 \%)$ & $95,773(64 \%)$ & $<0.001$ \\
\hline 5 yr survival & $299(72 \%)$ & $5,027(58 \%)$ & $4,531(63 \%)$ & $89,644(60 \%)$ & $<0.001$ \\
\hline 5 yr survival $(\mathrm{M})$ & $237(72 \%)$ & $3,602(58 \%)$ & $3,211(64 \%)$ & $66,686(61 \%)$ & $<0.001$ \\
\hline 5 yr survival $(F)$ & $64(73 \%)$ & $1,425(59 \%)$ & $1,094(66 \%)$ & $24,278(60 \%)$ & $<0.001$ \\
\hline
\end{tabular}

$\mathrm{AIP}=$ Asian Indian or Pakistani; $\mathrm{Ca}=$ cancer; $\mathrm{M}=$ male, $\mathrm{F}=$ female, $\mathrm{M}+\mathrm{F}=$ both sexes

Paradoxically, despite having similar amounts of surgery, radiation, or systemic therapy for bladder cancer, the US-AIP age-adjusted 5-year survival (overall, gender-specific, and stage-specific) was significantly better than the other three US ethnic groups examined. 


\subsection{Kidney Cancer}

Kidney cancer accounted for 364 (3\%) of all cancers in US-AIP in the SEER database from 1988-to-2008, similar to patients with kidney cancer in the other three US ethnic groups (3\% -to- $4 \%)$. However, more US-AIP with kidney cancer were men $(71 \%, \mathrm{p}<0.01)$, and the median age at diagnosis of kidney cancer was about a decade younger both in US-AIP men (54 years, $p<0.01)$ and in US-AIP women ( 58 years, $p<0.01)$ as compared to patients in the other three ethnic groups.

Table 2. Kidney cancer epidemiology \& survival in US-Asian Indians and Pakistanis (US-AIP) versus other US ethnic groups reported in SEER 1988-2008. Data are presented as number (\%) unless otherwise stated

\begin{tabular}{|c|c|c|c|c|c|}
\hline Variables & US-AIP & US-African & US-Hispanic & US-White & $P$-value \\
\hline SEER Total Cancers & 14,006 & 287,533 & 227,784 & $3,745,846$ & $N A$ \\
\hline KIDNEY Cancer & $364(3 \%)$ & $9,488(3 \%)$ & $7,061(3 \%)$ & $142,342(4 \%)$ & $P=0.65$ \\
\hline Incidence $/ 100,000$ & 9 & 21 & 18 & 19 & $<0.01$ \\
\hline Males & $258(71 \%)$ & $6,167(65 \%)$ & $4,449(63 \%)$ & $88,252(62 \%)$ & $<0.01$ \\
\hline \multicolumn{6}{|l|}{$\mathrm{M}+\mathrm{F}$ Ages } \\
\hline median, yrs & 55 & 65 & 66 & 64 & \multirow[t]{3}{*}{$<0.001$} \\
\hline 25th quartile, yrs & 45 & 55 & 56 & 54 & \\
\hline $75^{\text {th }}$ quartile, yrs & 66 & 76 & 75 & 74 & \\
\hline \multicolumn{6}{|l|}{ Male (M) Ages } \\
\hline median, yrs & 54 & 66 & 66 & 65 & \multirow[t]{3}{*}{$<0.01$} \\
\hline 25th quartile, yrs & 43 & 57 & 57 & 54 & \\
\hline 75th quartile, yrs & 65 & 76 & 75 & 76 & \\
\hline \multicolumn{6}{|l|}{ Female (F) Ages } \\
\hline median, yrs (F) & 58 & 64 & 65 & 62 & \multirow[t]{3}{*}{$<0.01$} \\
\hline 25th quartile, yrs & 47 & 55 & 66 & 53 & \\
\hline 75th quartile, yrs & 68 & 76 & 74 & 72 & \\
\hline Age $<65$ at diagnosis & $146(40 \%)$ & $2,849(30 \%)$ & $1,765(25 \%)$ & $39,860(28 \%)$ & $<0.001$ \\
\hline Grade I & $66(18 \%)$ & $2,468(26 \%)$ & $2,189(31 \%)$ & $42,719(30 \%)$ & $<0.001$ \\
\hline Grade II & $80(22 \%)$ & $3,325(35 \%)$ & $2,754(39 \%)$ & $48,412(34 \%)$ & $<0.001$ \\
\hline Grade III & $124(34 \%)$ & $2,375(25 \%)$ & $1,418(20 \%)$ & $29,896(21 \%)$ & $<0.001$ \\
\hline Grade IV & $94(26 \%)$ & $1,329(14 \%)$ & $712(10 \%)$ & $21,552(15 \%)$ & $<0.001$ \\
\hline Stage I & $66(18 \%)$ & $2,084(22 \%)$ & $1,909(27 \%)$ & $35,785(25 \%)$ & $<0.001$ \\
\hline Stage II & $95(26 \%)$ & $2,942(31 \%)$ & $2,681(38 \%)$ & $47,089(33 \%)$ & $<0.001$ \\
\hline Stage III & $161(44 \%)$ & $3,513(37 \%)$ & $2,045(29 \%)$ & $48,475(34 \%)$ & $<0.001$ \\
\hline Stage IV & $72(12 \%)$ & $951(10 \%)$ & $426(6 \%)$ & $11,487(8 \%)$ & $<0.001$ \\
\hline Surgery & $285(78 \%)$ & $7,590(80 \%)$ & $5,795(81 \%)$ & $115,629(81 \%)$ & $P=0.08$ \\
\hline Radiation & $138(38 \%)$ & $3,797(40 \%)$ & $2,548(37 \%)$ & $58,560(41 \%)$ & $P=0.06$ \\
\hline Systemic therapy & $298(82 \%)$ & $7,875(83 \%)$ & $5,937(84 \%)$ & $113,973(80 \%)$ & $P=0.14$ \\
\hline 1 yr survival & $318(87 \%)$ & $7,306(77 \%)$ & $5,942(84 \%)$ & $113,629(80 \%)$ & $<0.01$ \\
\hline 2 yr survival & $299(82 \%)$ & $6,739(71 \%)$ & $5,439(77 \%)$ & $105,527(74 \%)$ & $<0.01$ \\
\hline 3 yr survival & $284(78 \%)$ & $6,166(65 \%)$ & $5,161(73 \%)$ & $96,867(68 \%)$ & $<0.01$ \\
\hline $4 \mathrm{yr}$ survival & $263(72 \%)$ & $5,788(61 \%)$ & 4,867 (69\%) & $89,642(63 \%)$ & $<0.01$ \\
\hline $5 \mathrm{yr}$ survival & $259(71 \%)$ & $5,220(55 \%)$ & $4,580(65 \%)$ & $84,034(59 \%)$ & $<0.01$ \\
\hline 5 yr survival $(M)$ & $178(69 \%)$ & $3,337(54 \%)$ & $2,718(61 \%)$ & $51,191(58 \%)$ & $<0.001$ \\
\hline 5 yr survival $(F)$ & $76(72 \%)$ & $2,031(61 \%)$ & $1,753(67 \%)$ & $33,541(62 \%)$ & $<0.001$ \\
\hline
\end{tabular}

$\mathrm{AIP}=$ Asian Indian or Pakistani; $\mathrm{Ca}=$ cancer; $\mathrm{M}=$ male, $\mathrm{F}=$ female, $\mathrm{M}+\mathrm{F}=$ both sexes 
Also, the calculated incidence rate for kidney cancer in US-AIP appeared to be significantly lower ( 9 per 100,000; $\mathrm{p}<0.01)$ than the rates in African-Americans (21 per 100,000), US Hispanics (18 per 100,000), or US-Whites (19 per 100,000).

Again, although significantly more US-AIP were diagnosed with kidney cancers of higher grade and stage than patients in the other three ethnic groups, and they received similar levels or surgery, radiation, or systemic therapy, the US-AIP age-adjusted 5-year kidney cancer survival (overall, gender-specific, and stage-specific) was significantly better than the other three US ethnic groups, both for men $(\mathrm{p}<0.001)$ and for women $(\mathrm{p}<0.001)$

\subsection{Prostate Cancer}

Prostate cancer was documented in 2,086 US-AIP men (29\%) out of a total of 7,118 US-AIP men with cancer. This was comparable to the prostate cancer proportion in US-Hispanic men (32\%) and US White men (34\%), but lower than in African American men (38\%; $\mathrm{p}<0.001)$ [Table 3]. The calculated prostate cancer incidence rate of 83 per 100,000 in US-AIP men was significantly below the rate of 240 per 100,000 in African American men, 134 per 100,000 in US-Hispanic men, and 153 per 100,000 in US-White men $(p<0.001)$.

Table 3. Prostate cancer epidemiology \& survival in US-Asian Indians and Pakistanis (US-AIP) versus other US ethnic groups reported in SEER 1988-2008. Data are presented as number (\%) unless otherwise stated

\begin{tabular}{llllll}
\hline Variables & US-AIP & US-African & US-Hispanic & US-White & $P$-value \\
\hline SEER Total Male Cancers & 7,118 & 179,472 & 123,629 & $1,419,739$ & $N A$ \\
PROSTATE Ca & $2,086(29 \%)$ & $67,852(38 \%)$ & $39,856(32 \%)$ & $478,346(34 \%)$ & $<0.01$ \\
Incidence/100,000 & 83 & 240 & 134 & 153 & $<0.001$ \\
Male (M) Ages & & & & \\
median, yrs & 60 & 64 & 66 & 68 & $<0.001$ \\
25th quartile, yrs & 53 & 58 & 59 & 59 & \\
75th quartile, yrs & 69 & 71 & 74 & 77 & \\
Age $<$ 60 at Dx & $670(32 \%)$ & $18,972(28 \%)$ & $7,965(20 \%)$ & $105,436(22 \%)$ & $<0.001$ \\
Incr PSA at Dx & $1,732(78 \%)$ & $61,153(90 \%)$ & $35,173(88 \%)$ & $416,462(87 \%)$ & $<0.01$ \\
Abn DRE at Dx & $1,781(85 \%)$ & $52,873(78 \%)$ & $28,195(71 \%)$ & $344,810(72 \%)$ & $<0.01$ \\
Grade I & $63(3 \%)$ & $10,377(15 \%)$ & $4,481(11 \%)$ & $63,185(13 \%)$ & $<0.01$ \\
Grade II & $1,127(54 \%)$ & $40,131(59 \%)$ & $27,202(68 \%)$ & $311,926(65 \%)$ & $<0.01$ \\
Grade III & $878(42 \%)$ & $16,314(24 \%)$ & $7,996(20 \%)$ & $102,452(21 \%)$ & $<0.01$ \\
Grade IV & $18(1 \%)$ & $1,137(2 \%)$ & $427(1 \%)$ & $5,283(1 \%)$ & 0.21 \\
Stage I & $167(8 \%)$ & $8,262(12 \%)$ & $6,178(15 \%)$ & $67.968(14 \%)$ & $<0.001$ \\
Stage II & $501(24 \%)$ & $23,848(35 \%)$ & $16,042(40 \%)$ & $201,306(42 \%)$ & $<0.001$ \\
Stage III & $938(45 \%)$ & $23,919(35 \%)$ & $13,849(35 \%)$ & $149,287(31 \%)$ & $<0.001$ \\
Stage IV & $480(23 \%)$ & $12,309(18 \%)$ & $3,913(10 \%)$ & $63,285(13 \%)$ & $<0.001$ \\
Surgery & $879(42 \%)$ & $29,276(43 \%)$ & $15,878(40 \%)$ & $197,122(41 \%)$ & $P=0.17$ \\
Radiation & $461(22 \%)$ & $15,715(23 \%)$ & $8,429(21 \%)$ & $106,236(22 \%)$ & $P=0.14$ \\
Systemic therapy & $435(21 \%)$ & $15,801(23 \%)$ & $8,852(22 \%)$ & $107,037(22 \%)$ & $P=0.24$ \\
1 yr survival & $2,048(98 \%)$ & $61,842(91 \%)$ & $38,361(96 \%)$ & $455,430(95 \%)$ & $<0.01$ \\
2 yr survival & $1,985(95 \%)$ & $57,769(85 \%)$ & $35,972(90 \%)$ & $427,129(89 \%)$ & $<0.01$ \\
3 yr survival & $1,923(92 \%)$ & $54,891(81 \%)$ & $33,917(85 \%)$ & $399,812(84 \%)$ & $<0.01$ \\
4 yr survival & $1,836(88 \%)$ & $50,947(75 \%)$ & $31,984(80 \%)$ & $378,894(79 \%)$ & $<0.01$ \\
5 yr survival & $1,756(84 \%)$ & $48,325(71 \%)$ & $30,390(76 \%)$ & $355,177(74 \%)$ & $<0.01$ \\
\hline - A A & & & & \\
\end{tabular}

AIP = Asian Indian or Pakistani; $\mathrm{Ca}=$ cancer; $\mathrm{Dx}=$ diagnosis; $\mathrm{M}=$ male, $\mathrm{DRE}=$ digital rectal examination, $\mathrm{PSA}=$ Prostate specific antigen 
Prostate cancer in US-AIP men was found at a significantly earlier median age $(60 \mathrm{yrs} ; \mathrm{p}<0.001)$ than in African-American men (64 yrs), US-Hispanic men (66 yrs), or US-Caucasian men (68 yrs). Selecting the median US-AIP diagnosis age of 60 years as a "cut point", more US-AIP men with prostate cancer were below age 60 yrs $(34 \% ; p<0.001)$ when compared to men in the other three ethnic groups with prostate cancer. At diagnosis, fewer US-AIP men with prostate cancer had an elevated prostate specific antigen (PSA) blood level $(p<0.01)$, but paradoxically, more had an abnormal digital rectal examination (DRE) $(p<0.01)$ according to SEER data.

Again, although significantly more US-AIP men were diagnosed with prostate cancers of higher grade and stage than in the other three ethnic groups, and US-AIP men received similar levels of surgery, radiation, or systemic therapy, their age-adjusted 5-year prostate cancer survival (overall and stage-specific) was significantly better than for men with prostate cancer in the other three US ethnic groups $(\mathrm{p}<0.01)$.

\subsection{Multivariable Analysis}

For all four ethnic groups and all three genitourinary cancers studied, higher age at diagnosis, higher disease stage or grade, being divorced or widowed, and earlier era of diagnosis were linked to increased relative-risk (RR) of death.

For all four ethnic groups and all three genitourinary cancers studied, not having surgery or requiring either systemic therapy or radiation therapy were significantly associated with poorer survival and higher relative risk of death.

Of note, the SEER 2000-to-2008 data showed a significant decline in bladder, kidney, and prostate cancer relative risk of death, for all four ethnic groups studied, versus earlier era SEER 1994-to-2000 data.

Table 4. Relative risk (RR) of death in US-Asian Indians \& Pakistanis with prostate, kidney, or bladder cancer versus diagnostic variables (SEER 1988 - 2008)

\begin{tabular}{|c|c|c|c|c|c|c|}
\hline \multirow{2}{*}{ Cancer Type } & \multicolumn{2}{|c|}{ Prostate $\mathrm{N}=2,086$} & \multicolumn{2}{|c|}{ Kidney $N=364$} & \multicolumn{2}{|c|}{ Bladder $N=417$} \\
\hline & RR & $95 \% \mathrm{CI}$ & RR & $95 \% \mathrm{CI}$ & RR & $95 \% C I$ \\
\hline \multicolumn{7}{|c|}{ Age at diagnosis } \\
\hline$<50 \mathrm{yrs}$ & 1.09 & $1.02-1.18$ & 1.08 & $1.01-1.21$ & 1.06 & $0.95-1.19$ \\
\hline $51-60$ yrs* & 1.00 & & 1.00 & & 1.00 & \\
\hline $61-70$ yrs & 1.16 & $1.09-1.25$ & 1.27 & $1.17-1.38$ & 1.21 & $1.11-1.34$ \\
\hline $71-80 \mathrm{yrs}$ & 1.41 & $1.36-1.58$ & 1.59 & $1.46-1.69$ & 1.55 & $1.42-1.67$ \\
\hline$>81 \mathrm{yrs}$ & 1.89 & $1.79-1.97$ & 2.23 & $2.12-2.39$ & 2.29 & $2.19-2.38$ \\
\hline \multicolumn{7}{|l|}{ Marital status } \\
\hline Married* & 1.00 & & 1.00 & & 1.00 & \\
\hline Divorced & 1.31 & $1.22-1.39$ & 1.58 & $1.49-1.65$ & 1.66 & $1.53-1.76$ \\
\hline Widowed & 1.38 & $1.29-1.45$ & 1.62 & $1.48-1.69$ & 1.49 & $1.31-1.61$ \\
\hline \multicolumn{7}{|c|}{ Year of diagnosis } \\
\hline 1988-1994 & 1.57 & $1.48-1.70$ & 1.91 & $1.82-2.04$ & 1.88 & $1.72-1.99$ \\
\hline $1995-2000$ & 1.44 & $1.31-1.52$ & 1.21 & $1.14-1.35$ & 1.39 & $1.27-1.48$ \\
\hline $2000-2008^{*}$ & 1.00 & & 1.00 & & 1.00 & \\
\hline \multicolumn{7}{|c|}{ Stage at diagnosis } \\
\hline Stage I & 0.60 & $0.51-0.71$ & 0.78 & $0.64-0.86$ & 0.66 & $0.58-0.74$ \\
\hline Stage II* & 1.00 & & 1.00 & & 1.00 & \\
\hline Stage III & 1.96 & $1.83-2.07$ & 2.28 & $2.13-2.37$ & 1.98 & $1.86-2.09$ \\
\hline Stage IV & 2.94 & $2.86-3.05$ & 3.11 & $2.98-3.24$ & 3.22 & $2.07-3.33$ \\
\hline \multicolumn{7}{|c|}{ Grade at diagnosis } \\
\hline Grade I & 0.46 & $0.35-0.55$ & 0.61 & $0.52-0.74$ & 0.70 & $0.58-0.84$ \\
\hline Grade II* & 1.00 & & 1.00 & & 1.00 & \\
\hline Grade III & 2.58 & $2.41-2.66$ & 2.21 & $2.13-2.38$ & 1.67 & $1.53-2.83$ \\
\hline Grade IV & 2.89 & $2.77-2.98$ & 3.91 & $3.81-4.03$ & 2.99 & $2.85-3.13$ \\
\hline
\end{tabular}




\begin{tabular}{lllllll}
\hline \multirow{2}{*}{ Cancer Type } & \multicolumn{2}{l}{ Prostate $\mathrm{N}=2,086$} & \multicolumn{2}{l}{ Kidney $\mathrm{N}=364$} & \multicolumn{2}{l}{ Bladder $N=417$} \\
\cline { 2 - 7 } & $\mathrm{RR}$ & $95 \% \mathrm{CI}$ & $\mathrm{RR}$ & $95 \% \mathrm{CI}$ & $\mathrm{RR}$ & $95 \%$ CI \\
\hline Treatment types & & & & & 1.00 & \\
Surgery* & 1.00 & & 1.00 & & 3.06 & $2.95-3.19$ \\
No surgery & 1.71 & $1.63-1.77$ & 3.67 & $3.51-79$ & 3.06 & $1.61-1.95$ \\
Systemic therapy & 2.66 & $2.58-2.79$ & 2.11 & $2.01-2.24$ & 1.79 & $1.88-2.19$ \\
Radiation therapy & 1.96 & $1.89-2.05$ & 2.33 & $2.18-2.49$ & 2.01 & \\
\hline
\end{tabular}

* Referent category (in bold)

CI: confidence intervals

Relative Risk (RR): Calculated using Cox proportional hazards regression (SAS PHREG)

Table 5. Relative risk (RR) of death in African Americans with prostate, kidney, or bladder cancer versus diagnostic variables (SEER 1988 - 2008)

\begin{tabular}{|c|c|c|c|c|c|c|}
\hline \multirow{2}{*}{ Cancer Type } & \multicolumn{2}{|c|}{ Prostate $\mathrm{N}=41,664$} & \multicolumn{2}{|c|}{ Kidney $\mathrm{N}=5,672$} & \multicolumn{2}{|c|}{ Bladder $N=6,187$} \\
\hline & RR & $95 \% \mathrm{CI}$ & $\mathrm{RR}$ & $95 \% \mathrm{CI}$ & RR & $95 \% C I$ \\
\hline \multicolumn{7}{|l|}{ Age at diagnosis } \\
\hline$<50 \mathrm{yrs}$ & 0.92 & $0.87-0.95$ & 0.82 & $0.77-0.89$ & 0.89 & $0.84-0.95$ \\
\hline $51-60 \mathrm{yrs}^{*}$ & 1.00 & & 1.00 & & 1.00 & \\
\hline $61-70$ yrs & 1.37 & $1.34-1.40$ & 1.31 & $1.29-1.36$ & 1.35 & $1.29-1.41$ \\
\hline $71-80 \mathrm{yrs}$ & 1.88 & $1.81-1.92$ & 1.77 & $1.69-1.85$ & 1.85 & $1.79-1.93$ \\
\hline$>81 \mathrm{yrs}$ & 2.36 & $2.31-2.42$ & 2.42 & $2.35-2.50$ & 2.44 & $2.41-2.49$ \\
\hline \multicolumn{7}{|l|}{ Marital status } \\
\hline Married* & 1.00 & & 1.00 & & 1.00 & \\
\hline Divorced & 1.55 & $1.49-1.59$ & 1.45 & $1.38-1.52$ & 1.67 & $1.62-1.71$ \\
\hline Widowed & 1.74 & $1.70-1.79$ & 1.73 & $1.65-1.81$ & 1.88 & $1.82-1.93$ \\
\hline \multicolumn{7}{|l|}{ Year of diagnosis } \\
\hline $1988-1994$ & 2.06 & $1.99-2.09$ & 1.89 & $1.81-1.96$ & 2.25 & $2.18-2.35$ \\
\hline $1995-2000$ & 1.67 & $1.63-1.71$ & 1.53 & $1.49-1.60$ & 1.71 & $1.67-1.78$ \\
\hline $2000-2008^{*}$ & 1.00 & & 1.00 & & 1.00 & \\
\hline \multicolumn{7}{|l|}{ Stage at diagnosis } \\
\hline Stage I & 0.87 & $0.83-0.92$ & 0.88 & $0.79-0.95$ & 0.99 & $0.95-1.03$ \\
\hline Stage II* & 1.00 & & 1.00 & & 1.00 & \\
\hline Stage III & 2.05 & $1.99-2.09$ & 1.85 & $1.77-1.94$ & 2.18 & $2.11-2.26$ \\
\hline Stage IV & 2.94 & $2.87-3.01$ & 2.68 & $2.59-2.74$ & 3.29 & $3.16-3.37$ \\
\hline \multicolumn{7}{|l|}{ Grade at diagnosis } \\
\hline Grade I & 0.60 & $0.58-0.64$ & 0.67 & $0.59-0.76$ & 0.84 & $0.77-0.94$ \\
\hline Grade II* & 1.00 & & 1.00 & & 1.00 & \\
\hline Grade III & 1.96 & $1.89-2.00$ & 1.98 & $1.89-2.05$ & 1.78 & $1.68-1.85$ \\
\hline Grade IV & 2.65 & $2.59-2.71$ & 2.78 & $2.71-2.85$ & 2.57 & $2.51-2.63$ \\
\hline \multicolumn{7}{|l|}{ Treatment types } \\
\hline Surgery* & 1.00 & & 1.00 & & 1.00 & \\
\hline No surgery & 1.81 & $1.77-1.86$ & 2.66 & $2.58-2.76$ & 2.37 & $2.32-2.44$ \\
\hline Systemic therapy & 2.55 & $2.49-2.61$ & 2.03 & $1.96-2.11$ & 1.89 & $1.81-1.94$ \\
\hline Radiation therapy & 1.83 & $1.79-1.90$ & 1.78 & $1.70-1.85$ & 1.78 & $1.72-1.85$ \\
\hline
\end{tabular}

* Referent category (in bold)

CI: confidence intervals

Relative Risk (RR): Calculated using Cox proportional hazards regression (SAS PHREG) 
Table 6. Relative risk (RR) of death in US-Hispanics with prostate, kidney, or bladder cancer versus diagnostic variables (SEER 1988 - 2008)

\begin{tabular}{|c|c|c|c|c|c|c|}
\hline \multirow{2}{*}{ Cancer Type* } & \multicolumn{2}{|c|}{ Prostate $\mathrm{N}=39,856$} & \multicolumn{2}{|c|}{ Kidney $N=4,926$} & \multicolumn{2}{|c|}{ Bladder $N=4,729$} \\
\hline & $\mathrm{RR}$ & $95 \% \mathrm{CI}$ & $\mathrm{RR}$ & $95 \% \mathrm{CI}$ & $\mathrm{RR}$ & $95 \% C I$ \\
\hline \multicolumn{7}{|l|}{ Age at diagnosis } \\
\hline$<50 \mathrm{yrs}$ & 1.07 & $0.98-1.15$ & 1.01 & $0.97-1.05$ & 0.92 & $0.88-0.97$ \\
\hline $51-60$ yrs* & 1.00 & & 1.00 & & 1.00 & \\
\hline $61-70 \mathrm{yrs}$ & 1.26 & $1.22-1.30$ & 1.22 & $1.17-1.26$ & 1.36 & $1.29-1.40$ \\
\hline $71-80$ yrs & 1.63 & $1.58-1.68$ & 1.53 & $1.46-1.60$ & 1.67 & $161-1.72$ \\
\hline$>81 \mathrm{yrs}$ & 1.91 & $1.87-1.94$ & 2.02 & $1.96-2.09$ & 2.05 & $1.97-2.10$ \\
\hline \multicolumn{7}{|l|}{ Marital status } \\
\hline Married* & 1.00 & & 1.00 & & 1.00 & \\
\hline Divorced & 1.26 & $1.22-1.31$ & 1.54 & $1.49-1.58$ & 1.54 & $1.49-1.60$ \\
\hline Widowed & 1.31 & $1.27-1.35$ & 1.72 & $1.67-1.80$ & 1.50 & $1.47-1.54$ \\
\hline \multicolumn{7}{|l|}{ Year of diagnosis } \\
\hline 1988-1994 & 1.76 & $1.71-1.80$ & 1.73 & $1.68-1.79$ & 1.79 & $1.72-1.85$ \\
\hline $1995-2000$ & 1.32 & $1.28-1.38$ & 1.32 & $1.28-1.35$ & 1.35 & $1.30-1.42$ \\
\hline $2000-2008^{*}$ & 1.00 & & 1.00 & & 1.00 & \\
\hline \multicolumn{7}{|l|}{ Stage at diagnosis } \\
\hline Stage I & 0.52 & $0.47-0.60$ & 0.81 & $0.77-0.85$ & 0.72 & $0.67-0.78$ \\
\hline Stage II* & 1.00 & & 1.00 & & 1.00 & \\
\hline Stage III & 2.05 & $1.97-2.11$ & 2.18 & $2.13-2.23$ & 1.88 & $1.83-1.95$ \\
\hline Stage IV & 3.02 & $2.96-3.09$ & 2.91 & $2.86-2.97$ & 3.19 & $3.10-3.27$ \\
\hline \multicolumn{7}{|l|}{ Grade at diagnosis } \\
\hline Grade I & 0.45 & $0.39-0.50$ & 0.61 & $0.56-0.66$ & 0.66 & $0.58-0.75$ \\
\hline Grade II* & 1.00 & & 1.00 & & 1.00 & \\
\hline Grade III & 2.58 & $2.51-2.63$ & 2.01 & $1.95-2.09$ & 1.58 & $1.52-1.64$ \\
\hline Grade IV & 2.86 & $2.79-3.91$ & 3.87 & $3.78-3.97$ & 2.87 & $2.79-2.94$ \\
\hline \multicolumn{7}{|l|}{ Treatment types } \\
\hline Surgery* & 1.00 & & 1.00 & & 1.00 & \\
\hline No surgery & 1.70 & $1.64-1.76$ & 2.89 & $2.80-2.95$ & 2.95 & $2.88-3.05$ \\
\hline Systemic therapy & 2.54 & $2.51-2.58$ & 2.26 & $2.21-2.30$ & 1.86 & $1.81-1.92$ \\
\hline Radiation therapy & 1.79 & $1.76-1.84$ & 1.97 & $1.92-2.04$ & 2.17 & $2.11-2.25$ \\
\hline
\end{tabular}

* Referent category (in bold)

CI: confidence intervals

Relative Risk (RR): Calculated using Cox proportional hazards regression (SAS PHREG) 
Table 7. Relative risk (RR) of death in US-Whites/Caucasians with prostate, kidney, or bladder cancer versus diagnostic variables (SEER $1988-2008$ )

\begin{tabular}{|c|c|c|c|c|c|c|}
\hline \multirow{2}{*}{ Cancer Type } & \multicolumn{2}{|c|}{ Prostate $\mathrm{N}=278,346$} & \multicolumn{2}{|c|}{ Kidney $\mathrm{N}=53,846$} & \multicolumn{2}{|c|}{ Bladder $N=109,756$} \\
\hline & RR & $95 \% \mathrm{CI}$ & $\mathrm{RR}$ & $95 \% \mathrm{CI}$ & RR & $95 \% C I$ \\
\hline \multicolumn{7}{|l|}{ Age at diagnosis } \\
\hline$<50 \mathrm{yrs}$ & 0.96 & $0.94-0.97$ & 1.06 & $1.02-1.10$ & 1.03 & $0.99-1.07$ \\
\hline $51-60$ yrs* & 1.00 & & 1.00 & & 1.00 & \\
\hline $61-70 \mathrm{yrs}$ & 1.19 & $1.16-1.23$ & 1.25 & $1.21-1.29$ & 1.41 & $1.39-1.45$ \\
\hline $71-80 \mathrm{yrs}$ & 1.53 & $1.49-1.56$ & 1.59 & $1.56-1.62$ & 1.71 & $1.67-1.75$ \\
\hline$>81 \mathrm{yrs}$ & 1.85 & $1.82-1.89$ & 2.12 & $2.08-2.15$ & 2.56 & $2.49-2.61$ \\
\hline \multicolumn{7}{|l|}{ Marital status } \\
\hline Married* & 1.00 & & 1.00 & & 1.00 & \\
\hline Divorced & 1.29 & $1.24-1.32$ & 1.51 & $1.47-1.55$ & 1.47 & $1.42-1.52$ \\
\hline Widowed & 1.37 & $1.34-1.41$ & 1.65 & $1.62-1.69$ & 1.52 & $1.48-1.57$ \\
\hline \multicolumn{7}{|l|}{ Year of diagnosis } \\
\hline 1988-1994 & 1.81 & $1.78-1.84$ & 1.59 & $1.54-1.63$ & 2.05 & $1.99-2.10$ \\
\hline $1995-2000$ & 1.27 & $1.24-1.30$ & 1.22 & $1.18-1.27$ & 1.53 & $1.49-1.58$ \\
\hline 2000-2008* & 1.00 & & 1.00 & & 1.00 & \\
\hline \multicolumn{7}{|l|}{ Stage at diagnosis } \\
\hline Stage I & 0.74 & $0.72-0.75$ & 0.79 & $0.74-0.83$ & 0.84 & $0.80-0.89$ \\
\hline Stage II* & 1.00 & & 1.00 & & 1.00 & \\
\hline Stage III & 1.94 & $1.89-1.98$ & 1.94 & $1.89-2.00$ & 1.91 & $1.87-1.95$ \\
\hline Stage IV & 2.74 & $2.71-2.78$ & 2.75 & $2.71-2.80$ & 3.07 & $3.03-3.12$ \\
\hline \multicolumn{7}{|l|}{ Grade at diagnosis } \\
\hline Grade I & 0.61 & $0.58-0.65$ & 0.71 & $0.67-0.74$ & 0.75 & $0.72-0.79$ \\
\hline Grade II* & 1.00 & & 1.00 & & 1.00 & \\
\hline Grade III & 1.88 & $1.83-1.92$ & 2.05 & $1.98-2.11$ & 1.61 & $1.58-1.65$ \\
\hline Grade IV & 2.49 & $2.46-2.52$ & 3.54 & $3.49-3.59$ & 2.80 & $2.76-2.85$ \\
\hline \multicolumn{7}{|l|}{ Treatment types } \\
\hline Surgery* & 1.00 & & 1.00 & & 1.00 & \\
\hline No surgery & 1.76 & $1.72-1.79$ & 2.80 & $2.76-2.86$ & 2.66 & $2.61-2.69$ \\
\hline Systemic therapy & 2.70 & $2.66-2.74$ & 2.16 & $2.07-2.25$ & 1.79 & $1.75-1.82$ \\
\hline Radiation therapy & 1.90 & $1.86-1.94$ & 1.88 & $1.84-2.92$ & 2.07 & $2.01-2.13$ \\
\hline
\end{tabular}

* Referent category (in bold)

CI: confidence intervals

Relative Risk (RR): Calculated using Cox proportional hazards regression (SAS PHREG)

\section{Discussion}

SEER 1988-to-2008 data, released in May 2011, contains an interesting "survival disconnect" for three genitourinary cancers in US-Asian Indians and Pakistanis (AIP) as compared to African Americans, US Hispanics, and US Whites / Caucasians. All three US-AIP GU cancers had worse prognostic features at diagnosis, but better age-adjusted survival when compared to the other three US ethnic groups -- even though US-AIP appeared to have received similar treatment. This "survival disconnect" occurred despite all four ethnic groups and all three cancers studied having increasing age, disease stage, and grade associated with a rising relative risk (RR) of death, as one might expect.

What cultural, epidemiologic, or biologic differences might account for this better-than-expected US-AIP patient survival?

Asian Indians and Pakistanis (AIP) are closely related ethnic groups and together comprise about one-fifth of the entire world population. Asian Americans are defined by the US Census Bureau as “....individuals with origins in 
any of the original people of the Far East, Southeast Asia, or the Indian subcontinent" (Hossain et al., 2008). According to the year 2010 US census data, approximately 15 million Asians now reside in the US, $4.8 \%$ of the total USA population. Of these, 22\% are Chinese, 17\% Filipino, 12\% Asian Indian, 12\% Middle Eastern, 11\% Japanese, $10 \%$ Korean, $8 \%$ Pakistani, and $8 \%$ Vietnamese. There are now more than 3 million Asian Indians and Pakistanis in the United States, a number expected to double by 2040 (World health organization, 2011; US Citizen and..., 2011).

The SEER Program of the US National Cancer Institute has tracked cancer incidence and survival in the US by collecting data from population-based cancer registries that cover more than $26 \%$ of the population, including minority groups. SEER now covers $23 \%$ of African-Americans, $40 \%$ of Hispanics, $42 \%$ of American Indians and Alaskan Natives, 53\% of Asians, and 70\% of Hawaiian/Pacific Islanders (Goggins et al., 2007; Peters et al., 2008).

Knowledge of GU cancer risk factors in US-AIPs and in other Asian ethnic groups is lower than that of US-Whites (Peters, 2008). The rate of screening mammography, colonoscopy, and digital rectal examination is also lower in US-AIPs than US-Caucasians. Even the rate of wellness checkups is lower in US-AIPs below 18 years of age (Jian et al., 2005; Kolonel et al., 2000).

However, US-AIP may have a post diagnosis cancer survival advantage because of higher educational levels and socioeconomic status, as well as better insurance coverage. Educational levels are higher in US-AIPs than in other Asian or US ethnic groups. Although $48 \%$ of the overall Asian population achieves a bachelor's degree, more than $78 \%$ of US-AIPs attain this degree (Gloeckler et al., 2003). The median family income for all US-Asians in 2010 was $\$ 61,576$, but was $\$ 76,859$ for US Asian-Indians and $\$ 62,312$ for US-Pakistanis. US-AIPs have the highest level of health insurance coverage (93\%) compared to other ethnic groups in the US. Thus, higher household income and better health insurance coverage in US-AIP may provide an advantage in accessing health care (US Citizen and Immigration Services, 2010; Linn et al., 2002; Rastogi et al., 2008).

Environment or screening might contribute to differences in incidence or behavior of genitourinary cancers as US-AIP migrate from Asia to the west. For example, prostate cancer in the Indian subcontinent in 2008 was only the 4th leading cancer in Pakistani and Indian men (4-6\% of all cancers) according to the World Health Organization (Hossain et al., 2008; Jian et al., 2005), but was the most common cancer in US-AIP men (29\% of cancers). Factors known to influence genitourinary cancer risk include diet (e.g. vitamins, antioxidants, and saturated fat content), obesity, exercise, smoking, alcohol consumption, carcinogen exposure (e.g. arsenic in drinking water, radiation), education (knowledge \& attitudes), socioeconomic status, geographic location, and access to health care (Chan et al., 1998; Chan et al., 2001; Kolonel et al.; 1999; Pienta et al., 1993).

According to SEER data, US-AIP urologic cancers presented at significantly younger ages than in the other three comparison ethnic groups. Younger age at diagnosis could suggest fewer comorbidities and better general health, conferring a survival advantage. However, the survival data presented here were age-adjusted, so relative youth at diagnosis should not explain the better survival observed for US-AIP.

Younger US-AIPs readily adopt western lifestyles. Saturated fat intake, alcohol and tobacco consumption are higher in US-AIPs than in their counterparts in India and Pakistan (Chan et al., 2001; Kolonel et al., 1999; Hosain et al., 2012). The intake of tofu and leafy vegetables among US-AIPs is significantly less than the intake reported in recent studies in India and Pakistan. Also, US-AIP intake of curcumin is known to be less than in India and Pakistan (Cohen et al., 2000; Giovannucci et al., 1993). Although fruit intake might be comparable, overall vegetable intake of US-AIP women is significantly less than in India and Pakistan. Obesity is less frequent in US-AIP women than in other major US ethnic groups, but is more frequent than in non-immigrant AIP women (Lee et al., 2003; Ranasinghe et al., 2012). There is a known link of prostate, kidney, and bladder cancers with obesity and high fat intake (Rodriguez et al., 2007). In theory, a time lag in US-AIP immigrants adopting western diets and lifestyle might be reflected in different presentation and growth of their malignancies.

Genitourinary cancers in US-AIP may have subtle differences in biologic characteristics and behavior as compared to cancers in other groups. For example, although the SEER prostate cancer incidence in US-AIP was lower than in the other three ethnic groups, the cancer diagnosis occurred at a significantly younger age, with more than a third of men below 60 years of age. According to SEER, more US-AIPs with prostate cancer had an abnormal digital rectal examination (DRE), which usually implies more advanced disease and late screening or detection. Yet, fewer US-AIP had an elevated prostate specific antigen at diagnosis, which usually implies earlier detection and less advanced disease (Sinha et al., 2003; Yee et al., 2011). Perhaps differences in sexual practices, carcinogen exposure, or hormonal environment may impact the clinical behavior of prostate cancers in US-AIP men. Recent studies have investigated the anti-inflammatory or biologic effects of omega-3 fatty acids (Fradet et al., 2009; Sugimoto et al., 2012), TMPRSS2-ETS gene fusions (Narod et al., 2008), and XML RNA tumor virus latency 
(Dong et al., 2007; Klein et al., 2008), in US men with prostate cancer. It might be useful to know if any of these are different in US-AIP men with prostate cancer. Molecular characterization of US-AIP prostate cancers may yield useful biologic insights, and similar considerations might also apply to US-AIP patients with bladder or kidney cancers (Oakley-Girvan et al., 2003; Young et al., 1984; Maringe et al., 2012).

There are limitations to the SEER May 2011 data release reported here. The SEER database has no population denominator, so incidence rates had to be calculated based on US 2010 census data. Cancer rates among Asian-American populations have been difficult to measure since they represent a small and very diverse fraction of the US population. Despite this, the SEER 2011 data suggest that further study of US-AIP and other minority groups might be fruitful for further elucidating genitourinary tumor biology.

\section{Conclusion}

SEER 1988-to-2008 data contained a "survival disconnect" for genitourinary cancers in US-AIP. All three US-AIP GU cancers had worse prognostic features but better age-adjusted survival when compared to three other US ethnic groups, even though US-AIP appeared to have had similar treatment. Of note, SEER 2000-to-2008 data showed a significant decline in bladder, kidney, and prostate cancer relative risk of death for all four ethnic groups studied versus earlier era SEER 1994-to-2000 data.

\section{References}

Chan, J., Giovannucci, E., Andersson, S., Yuen, J., Adami, H., \& Wolk, A. (1998). Dairy products, calcium, phosphorous, vitamin d, and risk of prostate cancer (Sweden). Cancer Causes Control, 9(6), 559-566. http://dx.doi.org/10.1023/A:1008823601897

Chan, J., Stampfer, M., Ma, J., Gann, P., Gaziano, J., \& Giovannucci, E. (2001). Dairy products, calcium, and prostate cancer risk in the physicians' health study. American Journal of Clinical Nutrition, 74(4), 549-554. cebp.aacrjournals.org/content/12/7/597.full

Cohen, J., Kristal, A., \& Stanford, J. (2000). Fruit and vegetable intakes and prostate cancer risk. National Cancer Institute, 92(1), 61-68. http://dx.doi.org/10.1093/jnci/92.1.61

Dong, B., Kim, S., \& Hong, S. (2007). An infectious retrovirus susceptible to an ifn antiviral pathway from human prostate tumors. Proceedings and National Academy of Science USA, 104(5), 1655-1660. http://dx.doi.org/10.1073/pnas.0610291104

Fradet, V., Cheng, I., Casey, G., \& Witte, J. (2009). Dietary omega-3 fatty acids, cycleexygenase-2 generic variation, and aggressive prostate cancer risk. Clinical Cancer Research, 15(7), 2559-2566. http://dx.doi.org/10.1158/1078-0432.CCR-08-2503

Giovannucci, E., Rimm, E., \& Colditz, G., Stampfer, M. J., Ascherio, A., Chute, C. C., \& Willett, W. C. (1993). A prospective study of dietary fat and risk of prostate cancer. Journal of National Cancer Institute, 85(19), 1571-1579. http://dx.doi.org/10.1093/jnci/85.19.1571

Goggins, W., \& Wong, G. (2007). Poor survival for us pacific islander cancer patients: evidence from the surveillance, epidemiology, and end results database. American Journal of Clinical Nutrition, 25(36), 5738-5741. http://www.ahrq.gov/research/iomracereport/reldata2b.htm

Gloeckler Ries, L., Reichman, M., Lewis, D., Hankey, B., \& Edwards, B. (2003). Cancer survival and incidence from the surveillance, epidemiology, and end results (seer) program. Oncologist, 8(6), 541-552. http://dx.doi.org/10.1634/theoncologist.8-6-541

Hosain, G. M., Khan, M. M., Amiel, G. E., Lerner, S. P., Latini, D. M., \& Chen, G. J. (2012). Racial/ethnic differences in upper-tract urothelial cancer. Ethn Dis., 22(3), 295-301.

Hossain, A., Sehbai, A., Abraham, R., \& Abraham, J. (2008). Cancer health disparities among Indian and Pakistani immigrants in the United States: a surveillance, epidemiology, and end results-based study from 1998 to 2003. Cancer, 113(6), 1423-1430. http://dx.doi.org/10.1002/cncr.23686

Jani, A., Johnstone, P., Liauw, S., Master, V., \& Rossi, P. (2009). Prostate cancer modality time trent analyses from 1973 to 2004: a surveillance, epidemiology, and end results registry analysis. American Journal of Clinical Oncology, 33(2), 168-172.

Jian, L., Du, C., Lee, A., \& Binns, C. (2005). Do dietary lycopene and other carotenoids protect against prostate cancer? International Journal of Cancer, 113(6), 1010-1014. http://dx.doi.org/10.1002/ijc.20667

Klein, E., \& Silverman, R. (2008). Inflammation, infection, and prostate cancer. Curr Opin Urol, 18(3), 315-319. http://dx.doi.org/10.1097/MOU.0b013e3282f9b3b7 
Kolonel, L. N., Hankin, J. H., Whittemore, A. S., Wu, A. H., Gallagher, R. P., Wilkens, L. R., ... Paffenbarger, R. S. Jr. (2000). Vegetables, fruits, legumes and prostate cancer: a multiethnic case-control study. Cancer Epidemiol Biomarkers Prev., 9(8), 795-804. http://dx.doi.org/10.1093/jnci/91.5.414

Kolonel, L., Nomura, A., \& Cooney, R. (1999). Dietary fat and prostate cancer: current status. Journal of National Cancer Institute, 91(5), 414-428.

Lee, M., Gomez, S., Chang, J., Wey, M., Wang, R., \& Hsing, A. (2003). Soy and isoflavone consumption in relation to prostate cancer risk in china. Cancer Epidemiol Biomarkers Prevention, 12(7), 665-668.

Lin, S., Clarke, C., Prehn, A., Glaser, S., West, D., \& O'Malley, C. (2002). Survival differences among asian subpopulations in the united states after prostate, colorectal, breast, and cervical carcinomas. Cancer, 94(4), 1175-1182. http://dx.doi.org/10.1002/cncr.10319

Maringe, C., Mangtani, P., Rachet, B., Leon, D. A., Coleman, M. P., \& Silva, I. D. (2012). Cancer incidence in South Asian migrants to England, 1986-2004: Unravelling ethnic from socio-economic differentials. Int J Cancer. 2012 Sep 10. http://dx.doi.org/10.1002/ijc.27826

Narod, S., Seth, A., \& Nam, R. (2008). Fusion in the ets gene family and prostate cancer. British Journal of Cancer, 99(6), 847-851. http://dx.doi.org/10.1038/sj.bjc.6604558

Oakley-Girvan, I., Kolonel, L., Gallagher, R., Wu, A., Felberg, A., \& Whittemore, A. (2003). Stage at diagnosis and survival in a multiethnic cohort of prostate cancer patients. American Journal of Public Health, 93(10), 1753-1759. http://dx.doi.org/10.2105/AJPH.93.10.1753

Peinta, K., \& Esper, P. (1993). Risk factors for prostate cancer. Annals of Internal Medicine, 118(10), 793-803.

Peters, U., Littman, A., Kristal, A., Patterson, R., Potter, J., \& White, E. (2008). Vitamin e and selenium supplementation and risk of prostate cancer in the vitamins and lifestyle (vital) study cohort. Cancer Causes Control, 19(1), 75-87. http://dx.doi.org/10.1007/s10552-007-9072-y

Ranasinghe, W. K., De Silva, D., De Silva, M. V., Ranasinghe, T. I., Lawrentschuk, N., Bolton, D., \& Persad, R. (2012). Incidence of bladder cancer in sri lanka: analysis of the cancer registry data and review of the incidence of bladder cancer in the South asian population. Korean J Urol, 53(5), 304-9. Epub 2012 May 18. http://dx.doi.org/10.4111/kju.2012.53.5.304

Rodriguez, C., Freedland, S., \& Deka, A. (2007). Body mass index, weight change, and risk of prostate cancer in the cancer prevention study ii nutrition cohort. Cancer Epidemiol Biomarkers Prevention, 16(1), 63-69. http://dx.doi.org/10.1158/1055-9965.EPI-06-0754

SAS v 9.3 [computer program]. Version: 2011. www.sas.com

SEER*stat software V7.0.5 [computer program]. Version: September 2011. www.seer.cancer.gov

SEER(Surveillance, epidemiology and end results). (2011). Retrieved from http://www.seer.cancer.gov

Sinha, R., Anderson, D., McDonald, S., \& Greenwald, P. (2003). Cancer risk and diet in India. Journal of Postgrad Medicine, 49(3), 222-228.

Sugimoto, M., \& Kakehi, Y. (2012). Updated results from the European Randomized Study of Prostate-Specific Antigen (PSA) Screening for Prostate Cancer: are Asian countries encouraged to promote PSA screening? Asian J Androl. 2012 Jun 18. www.scibite.com/site/library/2012_7/1/0/22751444.html

US citizen and immigration services. (2011). Retrieved from http://www.uscis.gov

WHO (World health organization data and statistics). (2011). Retrieved from http://www.who.int

Yee, D. S., Ishill, N. M., Lowrance, W. T., Herr, H. W., \& Elkin, E. B. (2011). Ethnic differences in bladder cancer survival. Urology, 78(3), 544-9. http://dx.doi.org/10.1016/j.urology.2011.02.042

Young, J. J., Ries, L., \& Pollack, E. (1984). Cancer patient survival among ethnic groups in the united states. Journal of National Cancer Institute, 73(2), 341-352. 